\title{
Arrival Times in a Zero-Range Process with Injection and Decay
}

\author{
B. Hertz Shargel ${ }^{1}$, M. R. D’Orsogna ${ }^{2}$, T. Chou ${ }^{1,3}$ \\ ${ }^{1}$ Department of Mathematics, UCLA, Los Angeles, CA 90095-1555 \\ ${ }^{2}$ Department of Mathematics, CSUN, Los Angeles, CA 91330-8313 \\ ${ }^{3}$ Department of Biomathematics, UCLA, Los Angeles, CA 90095-1766
}

\begin{abstract}
Explicit expressions for arrival times of particles moving in a onedimensional Zero-Range Process (ZRP) are computed. Particles are fed into the ZRP from an injection site and can also evaporate from anywhere in the interior of the ZRP. Two dynamics are considered; bulk dynamics, where particle hopping and decay is proportional to the numqber of particles at each site, and surface dynamics, where only the top particle at each site can hop or evaporate. We find exact solutions in the bulk dynamics case and for a single-site ZRP obeying surface dynamics. For a multisite ZRP obeying surface dynamics, we compare simulations with approximations obtained from the steady-state limit, where mean interarrival times for both models are equivalent. Our results highlight the competition between injection and evaporation on the arrival times of particles to an absorbing site.

PACS numbers: 05.60.-k,87.16.Ac,05.10.Ln
\end{abstract}

\section{Introduction}

The Zero-Range Process (ZRP) is a stochastic model to describe the dynamics of far from equilibrium, interacting particles hopping between lattice sites [1, 2, 3. The ZRP has been used in many applications as a paradigm for transport processes, including traffic flows, shaken granular gases, network dynamics, phase separation, and particle condensation and clustering 4 . Mathematical interest also arises from the fact that a simple connection can be made between the ZRP and the totally asymmetric exclusion process (TASEP) [1] and that in certain cases - particularly for conserved systems exact factorisable steady-state solutions can be derived [5].

In this paper, we compute the multiple passage times of particles obeying ZRP dynamics to reach a final absorbing site. We treat a nonconserved system where particles are injected at the origin and evaporate as they drift right towards the end site of the lattice, as shown in Fig. 1. This type of dynamics may be applied to many specific micro-biological systems. For example, molecular motors may attach at one end of a microtubule, but desorb while traversing it. The distribution of arrival times of the motors will depend on their speed, and injection and desorption rates. Other examples include virus entry and transport to the nucleus, where the viral cargo is transported by molecular motors while being subject to degradation [6, and sperm entry into egg cells, where the first sperm to penetrate all layers of the cell triggers 

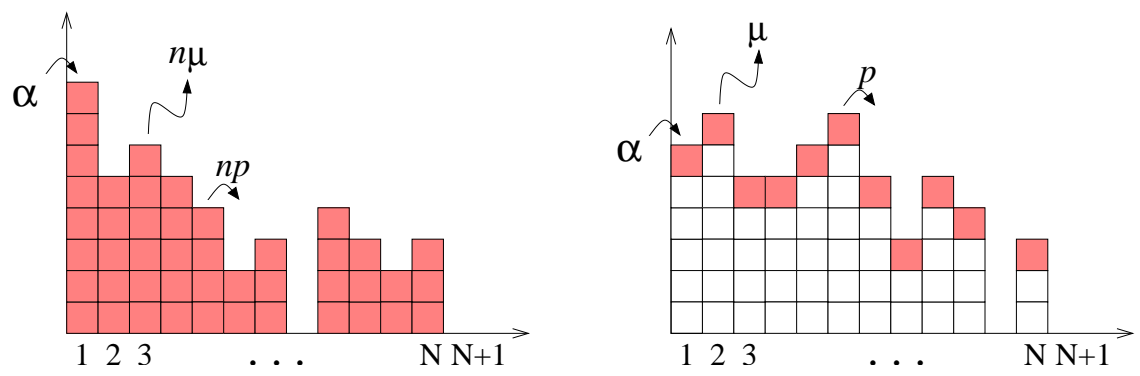

Figure 1. Two realisations of a Zero-Range Process. In (a), any one of the red particles in the bulk can hop to the right or decay, while in (b), only the surface particle (in red) in each pile can hop or decay, the underlying particles being protected by the top particle.

a block for subsequent ones 7. In all applications there is a flux of particles, or "immigration," into the first site as well as particle annihilation at every location along the microtubule or layer within the egg cell.

Unlike the first passage time (FPT) problem of a single, conserved particle undergoing a simple random walk, the first passage time of a nonconserved, multiparticle problem cannot be solved by analysing steady-states. In problems with injection and decay, the dynamics of particles reaching a specific "absorbing" site are complicated by subsequent particle arrivals, as well as arrival times conditioned on particles reaching the absorbing site. Since we will be concerned with a specific initial configuration, and wish to understand how the ZRP first reaches another configuration, we must find time-dependent solutions for the dynamics of the ZRP. Nonetheless, despite the nonconserved nature of the problem, steady-state solutions can still sometime provide useful approximations for FPTs of the ZRP in certain limits.

We present exact solutions for arrival times in a finite-sized ZRP obeying two specific dynamical rules. In the first case, which we denote as "bulk dynamics" and which is illustrated in Fig. 1(a), all particles at a site are equally likely to hop to the next site. In the second "surface dynamics" case, depicted in Figure 1(b), only one particle can hop to its neighboring site. These two cases are limits of the ZRP and may serve as a model system for many physical systems.

We begin our analysis in the following section with bulk dynamics. In this case, the particles are independent and we find exact analytic expressions for the distributions of the passage times for the $k^{\text {th }}$ particle to arrive at the absorbing site $N+1$. For mathematical completeness, we present two derivations of the solution. The first involves explicit enumeration of the number of particles injected, evaporated, and having reached the absorbing site by time $t$. The second involves writing down a Master equation, which is solved using generating functions and the method of characteristics. All results for the bulk case are exact. In the third section, we define a ZRP with surface dynamics, where only one particle at each site, if it exists, is allowed to hop or desorb. In this case, we can only find exact solutions for a single site ZRP. For a multisite ZRP, we find particle arrival times in certain limits using a steady-state approximation, and compare them with results derived from Monte Carlo simulations. 


\section{Zero-range Model with Bulk Dynamics}

In our bulk-dynamic ZRP, beginning at time $t=0$, particles are injected into the first site of an empty lattice whose positions are denoted by $\{1,2, \ldots, N+1\}$. The injection occurs as a Poisson process with rate $\alpha$. Each particle can then hop one site to the right with rate $p$ or evaporate with rate $\mu$, independently of others. The forward hopping and evaporation processes continue until the particle reaches site $N+1$ or is desorbed from the lattice. We wish to calculate the distribution of times for the $k^{\text {th }}$ particle to reach site $N+1$.

\subsection{Explicit enumeration of particle fates}

Denoting by $T_{k}$ the time at which the final absorbing site $N+1$ is reached for the $k^{\text {th }}$ time, we consider the accumulated number of hits $H(t)$ by time $t$ defined by $P(H(t)=k)=P\left(T_{k} \leq t<T_{k+1}\right)$. The primary result of this section is that $H(t)$ is Poisson distributed, with rate parameter

$$
\begin{aligned}
\lambda(t) & =\alpha\left(\frac{p}{\mu+p}\right)^{N}\left(t-\frac{N}{\mu+p}\right) \\
& -\frac{\alpha}{\mu+p} e^{-(\mu+p) t} \sum_{\ell=0}^{N-1}\left(\frac{p}{\mu+p}\right)^{\ell} \sum_{m=0}^{\ell}\left(\frac{\mu}{p+\mu} \sum_{i=0}^{m} \frac{((\mu+p) t)^{i}}{i !}-\frac{((\mu+p) t)^{m}}{m !}\right) .
\end{aligned}
$$

From this result we can find the survival probability $S_{k}(t)$ that the final site has been hit by $k-1$ or fewer particles,

$$
S_{k}(t)=\sum_{j=0}^{k-1} P(H(t)=j)=e^{-\lambda(t)} \sum_{j=0}^{k-1} \frac{\lambda(t)^{j}}{j !} .
$$

Similarly, the distribution of $T_{i}$ can be represented as the sum

$$
P\left(T_{i} \leq t\right)=\sum_{j=i}^{\infty} P(H(t)=j)=e^{-\lambda(t)} \sum_{j=i}^{\infty} \frac{\lambda(t)^{j}}{j !} .
$$

To derive the distribution of $H(t)$, we begin by noting that we may break up the event $\{H(t)=k\}$ according to how many particles $n$ were injected before time $t$, each with common probability $q(t)$ of reaching site $N+1$ by time $t$ (see Fig. 2). The probability that exactly $k$ of those $n$ particles reach site $N+1$ is Binomially distributed with parameter $q(t)$, so that

$$
\begin{aligned}
P(H(t)=k) & =\sum_{n=k}^{\infty} \frac{(\alpha t)^{n} e^{-\alpha t}}{n !} \frac{n !}{k !(n-k) !} q(t)^{k}(1-q(t))^{n-k} \\
& =\frac{q(t)^{k}(\alpha t)^{k}}{k !} e^{-\alpha t} \sum_{n=k}^{\infty} \frac{(\alpha(1-q(t)) t)^{n-k}}{(n-k) !} \\
& =\frac{(\alpha q(t) t)^{k}}{k !} e^{-\alpha q(t) t} .
\end{aligned}
$$




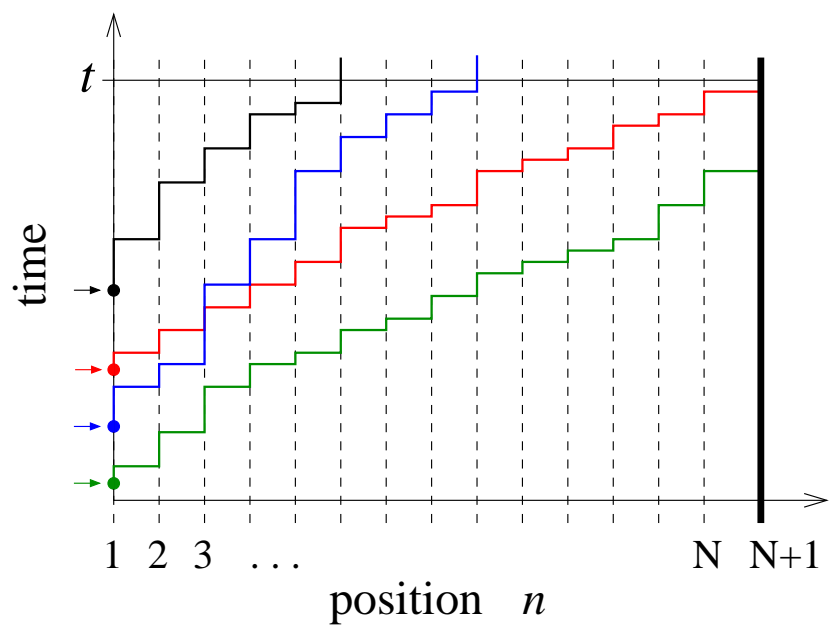

Figure 2. Space-time plot of ZRP bulk dynamics. In this realisation, 4 particles are injected by time $t$, and are the only ones capable of contributing to $H(t)$. Trajectories that intersect the vertical line have arrived at site $N+1$ within time $t$. Trajectories that intersect with the horizontal line at time $t$ are those that failed to reach the absorbing site $N+1$ by time $t$.

This implies that $H(t)$ is Poisson distributed with parameter

$$
\lambda(t)=\alpha q(t) t .
$$

Deriving the probability $q(t)$ is therefore our task. The particle injected at time $\tau$ is characterised by its decay time $\zeta_{\tau}$, which is exponentially distributed with mean $1 / \mu$, and by its arrival time $X_{\tau}$ at the target site $N+1$ excluding the possibility of decay. As this latter random variable is the sum of exponentials, it is $\operatorname{Gamma}(N, k)$-distributed 9. The probability $q(t)$ of reaching site $N+1$ is then given by the probability that the arrival time precedes both the chosen time limit $t$ or the time of decay, averaged over the possible injection times $\tau$. Symbolically,

$$
q(t)=\frac{1}{t} \int_{0}^{t}\left[P\left(X_{\tau} \leq t-\tau \leq \zeta_{\tau}\right)+P\left(X_{\tau} \leq \zeta_{\tau} \leq t-\tau\right)\right] \mathrm{d} \tau .
$$

Since $X_{\tau}$ and $\zeta_{\tau}$ are independent, the first probability $P\left(X_{\tau} \leq t-\tau \leq \zeta_{\tau}\right)=P\left(X_{\tau}<\right.$ $t-\tau) P\left(\zeta_{\tau}>t-\tau\right)$. And since $\zeta_{\tau}$ is exponentially distributed with mean $\mu$, and $X_{\tau}$ is Gamma-distributed, the first probability in the integrand of Eq.6 is simply

$$
\begin{aligned}
P\left(X_{\tau} \leq t-\tau \leq \zeta_{\tau}\right) & =F_{\Gamma}(t-\tau ; N, p)\left[1-F_{\zeta_{\tau}}(t-\tau)\right] \\
& =F_{\Gamma}(t-\tau ; N, p) e^{-\mu(t-\tau)}
\end{aligned}
$$

where $F_{\Gamma}(s ; N, \beta)$ is the Gamma distribution function. The second probability, and many of the computations to follow, rely on the following equivalent representations of this function,

$$
F_{\Gamma}(s ; N, \beta)=\frac{\gamma(N, \beta s)}{\Gamma(N)}=1-e^{-\beta s} \sum_{\ell=0}^{N-1} \frac{(\beta s)^{\ell}}{\ell !} .
$$


Here $\gamma(z, w)=\int_{0}^{w} t^{z-1} e^{-t} \mathrm{~d} t$ is the lower incomplete Gamma function, and the right hand equality with the Erlang distribution holds because $N$ is an integer [9]. Expression 8 leads to the following useful identities for $F_{\Gamma}$ :

$$
\begin{aligned}
\int_{0}^{s} F_{\Gamma}(u ; N, \beta) \mathrm{d} u & =\int_{0}^{s}\left(1-e^{-\beta u} \sum_{\ell=0}^{N-1} \frac{(\beta u)^{\ell}}{\ell !}\right) \mathrm{d} u=s-\sum_{\ell=0}^{N-1} \frac{1}{\ell !} \int_{0}^{s}(\beta u)^{\ell} e^{-\beta u} \mathrm{~d} u \\
& =s-\frac{1}{\beta} \sum_{\ell=0}^{N-1} \frac{\gamma(\ell+1, \beta s)}{\Gamma(\ell+1)} \equiv s-\frac{1}{\beta} \sum_{\ell=0}^{N-1} F_{\Gamma}(s ; \ell+1, \beta) .
\end{aligned}
$$

and

$$
\int_{0}^{s} e^{-\eta u} F_{\Gamma}(u ; N, \beta) \mathrm{d} u=\frac{1}{\eta}\left(1-e^{-\eta s}\right)-\frac{1}{\eta+\beta} \sum_{\ell=0}^{N-1}\left(\frac{\beta}{\eta+\beta}\right)^{\ell} F_{\Gamma}(s ; \ell+1, \eta+\beta) .
$$

Returning to the second probability in Eq.6, Eq.10 yields

$$
\begin{aligned}
P\left(X_{\tau} \leq \zeta_{\tau} \leq\right. & t-\tau)=\int_{0}^{t-\tau} \mu e^{-\mu s} F_{\Gamma}(s ; N, p) \mathrm{d} s \\
& =1-e^{-\mu(t-\tau)}-\frac{\mu}{\mu+p} \sum_{\ell=0}^{N-1}\left(\frac{p}{\mu+p}\right)^{\ell} F_{\Gamma}(t-\tau ; \ell+1, \mu+p),
\end{aligned}
$$

so that upon substituting Eqs. 7 and 11 into Eq.6] we obtain

$$
\begin{aligned}
q(t)=1+\frac{1}{t} \int_{0}^{t}[ & F_{\Gamma}(t-\tau, N, p) e^{-\mu(t-\tau)}-e^{-\mu(t-\tau)} \\
& \left.\quad-\frac{\mu}{\mu+p} \sum_{\ell=0}^{N-1}\left(\frac{p}{\mu+p}\right)^{\ell} F_{\Gamma}(t-\tau ; \ell+1, p+\mu)\right] d \tau .
\end{aligned}
$$

Evaluating the integral termwise using Eqs.9 and 10 we find

$q(t)=1-\frac{1}{t(\mu+p)} \sum_{\ell=0}^{N-1}\left(\frac{p}{\mu+p}\right)^{\ell}\left[\mu t+F_{\Gamma}(t ; \ell+1, \mu+p)-\frac{\mu}{\mu+p} \sum_{m=0}^{\ell} F_{\Gamma}(t ; m+1, \mu+p)\right]$.

We can now expand the distribution functions in terms of a finite sum. Performing some algebraic simplifications yields the closed form representation

$$
\begin{aligned}
q(t)=1-\frac{1}{t(\mu+p)} & \sum_{\ell=0}^{N-1}\left(\frac{p}{\mu+p}\right)^{\ell}\left[\frac{p-\ell \mu}{\mu+p}+\mu t+\right. \\
& \left.e^{-(\mu+p) t} \sum_{m=0}^{\ell}\left(\frac{\mu}{\mu+p} \sum_{i=0}^{m} \frac{((\mu+p) t)^{i}}{i !}-\frac{((\mu+p) t)^{m}}{m !}\right)\right] .
\end{aligned}
$$

Eq.12 may be further simplified by extracting the first two terms in the brackets from the sum and using the identity

$$
\sum_{\ell=1}^{n} \ell a^{\ell}=\frac{a\left(1-a^{n+1}\right)-(n+1)(1-a) a^{n+1}}{(1-a)^{2}},
$$




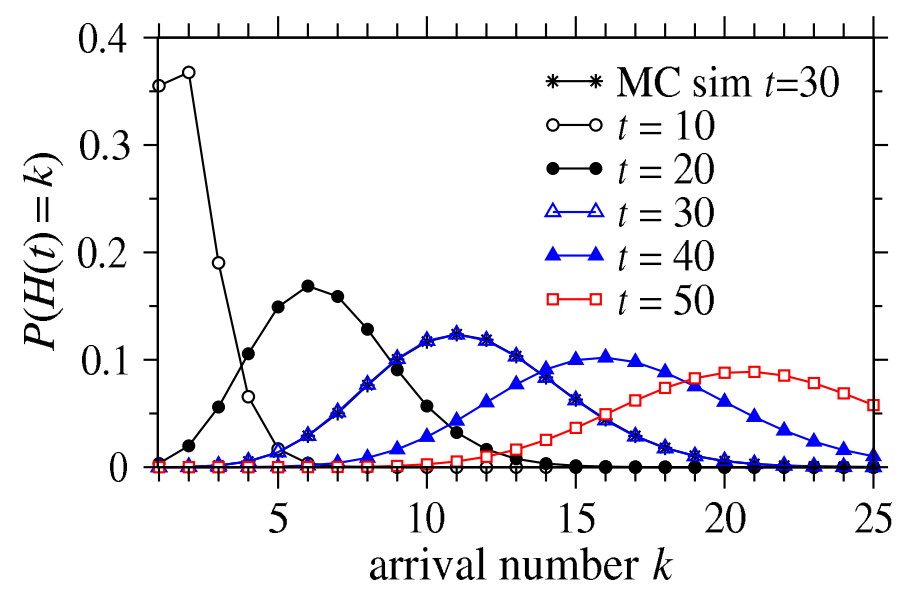

Figure 3. Plots of the distribution $P(H(t)=k)$ of the number of hits $H(t)$ that have occured up to time $t$. Parameters used were $p \equiv 1, N=10, \mu=0.2$, and $\alpha=3$. The distribution is plotted for times $t=5,10,20,30,40,50$. Monte Carlo simulation (300000 runs) was used to verify the results for $t=30$.

obtained by differentiating the well-known identity $\sum_{\ell=0}^{n} a^{\ell}=\left(1-a^{n+1}\right) /(1-a)$. We finally end up with

$$
\begin{aligned}
q(t) & =\left(\frac{p}{\mu+p}\right)^{N}\left(1-\frac{N}{t(\mu+p)}\right)-\frac{1}{t(\mu+p)} \\
& \times e^{-(\mu+p) t} \sum_{\ell=0}^{N-1}\left(\frac{p}{\mu+p}\right)^{\ell} \sum_{m=0}^{\ell}\left(\frac{\mu}{p+\mu} \sum_{i=0}^{m} \frac{((\mu+p) t)^{i}}{i !}-\frac{((\mu+p) t)^{m}}{m !}\right),
\end{aligned}
$$

which yields Eq.11, In Fig. 3, and in all subsequent plots, we nondimensionalise all rates in terms of $p$ and times in terms of $p^{-1}$. The distribution $P(H(t)=k)$ plotted in Fig. 3 shows that the probability of more arrivals increases with time after the start of injection increases. We compared and verified our results at time $t=30$ with a Monte Carlo simulation of the bulk dynamics using the Bortz-Kalos-Lebowitz algorithm [12. We performed 300000 runs, each with parameters $t=30, N=10, p=1, \mu=0.2$ and $\alpha=3$.

From the survival probability $S_{k}(t)$ found from Eq. 2. all moments $\sigma$ of the $k^{\text {th }}$ passage times can be computed,

$$
\left\langle T_{k}^{\sigma}\right\rangle=-\int_{0}^{\infty} t^{\sigma} \frac{\mathrm{d} S_{k}}{\mathrm{~d} t} \mathrm{~d} t
$$

The mean $(\sigma=1)$ first $(k=1)$ passage time to the absorbing site can be found from

$$
\begin{aligned}
& S_{1}(t)=P(H(t)=0)=\exp \left[\alpha\left(\frac{p}{\mu+p}\right)^{N}\left(\frac{N}{\mu+p}-t\right)\right. \\
& \left.+\frac{\alpha}{\mu+p} e^{-(\mu+p) t} \sum_{\ell=0}^{N-1}\left(\frac{p}{\mu+p}\right)^{\ell} \sum_{m=0}^{\ell}\left(\frac{\mu}{\mu+p} \sum_{i=0}^{m} \frac{((\mu+p) t)^{i}}{i !}-\frac{((\mu+p) t)^{m}}{m !}\right)\right]
\end{aligned}
$$

and Eq.15. An explicit expression can be found for a single-site ZRP $(N=1)$ : 


$$
\left\langle T_{1}\right\rangle=\frac{e^{x} x^{-x}}{\mu+p} \gamma(x, x), \quad N=1,
$$

where

$$
x \equiv \frac{\alpha p}{(\mu+p)^{2}} .
$$

Upon approximating the lower incomplete gamma function $\gamma(x, x) \equiv \Gamma(x)-\Gamma(x, x)$ in the $x \rightarrow 0$ limit, we find

$$
\left\langle T_{1}\right\rangle=\frac{1}{\mu+p}\left[\frac{1}{x}+1+\mathcal{O}(x)\right], \quad N=1 .
$$

In the $x \rightarrow \infty$ limit, we apply the method of steepest descents [16] to the integral definition of the $\Gamma$-function to find

$$
\left\langle T_{1}\right\rangle=\frac{1}{\mu+p} \sqrt{\frac{\pi}{2 x}}\left[1+\frac{12}{x}+\mathcal{O}\left(x^{-2}\right)\right], \quad N=1 .
$$

For $N>1$, the integral in Eq.15]does not have a simple representation in non-integral form, nor can the mean $k^{\text {th }}$ passage times be calculated explicitly in the $N=1$ case for $k>1$. However we can find some asymptotic results for large $k$ values in the "fast dynamics" limit. If we denote by $\tau$ the characteristic time $\tau=N /(\mu+p)$ for a particle just injected to reach the final site, and consider times $t$ such that $t \gg \tau$ Eq.1 may be written as

$$
\lambda(t)=\alpha_{\mathrm{eff}}(t-\tau)+\mathcal{O}\left(e^{-t(\mu+p)}\right),
$$

where $\alpha_{\mathrm{eff}}=\alpha p^{N}(\mu+p)^{-N}$ is an effective injection rate from the perspective of the final site that takes into account decay. Because $t \gg \tau$ holds for all but a negligible part of the range of the integral in Eq,15, we have

$$
\left\langle T_{k}\right\rangle=\frac{k}{\alpha_{\text {eff }}}+\tau+\mathcal{O}\left(\tau^{2}\right) .
$$

The assumption on $t$ translates onto a condition on $k$, so that Eq.22 remains valid as long as $\left\langle T_{k}\right\rangle \gg \tau$, or $k \gg \alpha_{\mathrm{eff}} \tau$.

In Fig. 4, we plot the interarrival times $\left\langle T_{k}\right\rangle-\left\langle T_{k-1}\right\rangle$ as a function of $k, \mu$, and $\alpha$. Fig. 4(a) shows that larger desorption rates permit the system to reach steadystate faster, where interarrival times approach the limit $1 / \alpha_{\text {eff }}$, for smaller values of $k$. In Fig. 4(b), we see that the mean interarrival times, including the mean first passage time, increase exponentially for large $\mu$. This result is different from that of the problem where only a single particle is injected, in which the conditional mean first passage time of that single particle decreases has desorption $\mu$ increases. Because the single particle problem needs to be conditioned on arrival, only very fast trajectories can survive the desorption process, leading to mean first passage times that decrease rapidly with increasing $\mu$. Finally, Fig. 4(c) plots the mean interarrival times as a function of injection rate $\alpha$.

In the next section we rederive the same results above by solving the corresponding Master equation using generating functions. Using this approach, we can not only recover the mean passage times to site $N+1$, but the full particle occupation distribution function $P\left(n_{1}, \ldots, n_{N+1}, t\right)$. 

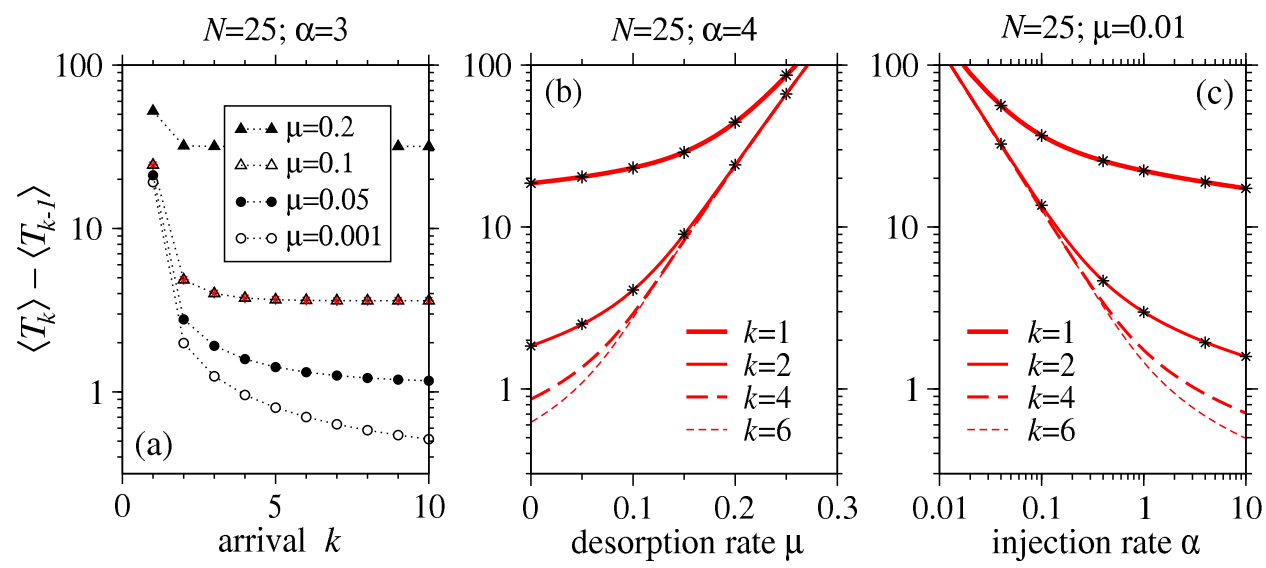

Figure 4. Interarrival times $\left\langle T_{k}\right\rangle-\left\langle T_{k-1}\right\rangle$ for the ZRP obeying bulk dynamics (with $p \equiv 1$ ). (a) Interarrival times as a function of arrival $k$ various values of the desorption rate $\mu$ for fixed $\alpha=3$. (b) Arrival times of the first, second, fourth, and sixth particles as a function of $\mu$ for fixed $\alpha=4$. (c) First, second, fourth, and sixth arrival times as a function of the injection rate $\alpha$ for $\mu=0.01$. All plots were evaluated using $N=25$. Results were verified using Monte-Carlo simulations (asterisks).

\subsection{Solving the bulk dynamics via generating functions}

In this section, for mathematical completeness, we rederive the survival probability using generating function methods applied to the Master equation for describing the probability $P\left(\left\{n_{\ell}\right\}, t\right)$ of having $\left\{n_{\ell}\right\}$ particles on each of the $1 \leq \ell \leq N+1$ sites:

$$
\begin{aligned}
\dot{P}\left(\left\{n_{\ell}\right\}, t\right)= & -\alpha\left[P\left(\left\{n_{\ell}\right\}, t\right)-P\left(n_{1}-1, \ldots, n_{N+1}, t\right)\left(1-\delta_{n_{1}, 0}\right)\right] \\
& -(\mu+p) \sum_{j=1}^{N} n_{j} P\left(\left\{n_{\ell}\right\}, t\right)+\mu \sum_{j=1}^{N}\left(n_{j}+1\right) P\left(n_{1}, \cdots, n_{j}+1, \cdots, t\right) \\
& +p \sum_{j=1}^{N}\left(n_{j}+1\right) P\left(n_{1}, \cdots, n_{j}+1, n_{j+1}-1 \cdots, t\right)\left(1-\delta_{n_{j+1}, 0}\right) .
\end{aligned}
$$

The survival probability $S_{1}(t)$ is defined by the probability of having no particles in the absorbing site

$$
S_{1}(t)=\sum_{n_{1}, n_{2} \cdots n_{N}} P\left(n_{1}, \cdots, n_{N+1}=0, t\right) .
$$

After setting $n_{N+1}=0$ in Eq.23 we consider the dynamics of the constrained generating function defined as

$$
G_{0}\left(z_{1}, \cdots, z_{N}, t\right)=\sum_{n_{1}, \cdots n_{N}=0}^{\infty} z_{1}^{n_{1}} \cdots z_{N}^{n_{N}} P\left(n_{1}, \cdots n_{N}, n_{N+1}=0, t\right) .
$$


Upon multiplying Eq.23 by $z_{1}^{n_{1}} \cdots z_{N}^{n_{N}}$ and summing over all possible values of $n_{j}$, $1 \leq j \leq N$, we find a first order partial differential equation for $G_{0}\left(z_{1}, \ldots, z_{N}, t\right)$ which we can solve using the method of characteristics [17. We find that $G_{0}$ obeys

$$
\frac{\mathrm{d} G_{0}}{\mathrm{~d} t}=\alpha\left(z_{1}-1\right) G_{0}
$$

along the trajectories defined by

$$
\begin{aligned}
\frac{\mathrm{d} z_{1}(t)}{\mathrm{d} t} & =(\mu+p) z_{1}(t)-\mu-p z_{2}(t), \\
\frac{\mathrm{d} z_{j}(t)}{\mathrm{d} t} & =(\mu+p) z_{j}(t)-\mu-p z_{j+1}(t), \\
\frac{\mathrm{d} z_{N}(t)}{\mathrm{d} t} & =(\mu+p) z_{N}(t)-\mu .
\end{aligned}
$$

The initial condition $P\left(n_{1}, \cdots, n_{N}, n_{N+1}=0, t=0\right)=\delta_{n_{1}, 0} \cdots \delta_{n_{N}, 0}$ gives $G_{0}\left(z_{0}, \cdots z_{N}, t=0\right)=1$. Equations 27 can be written in the form

$$
\frac{\mathrm{d} \mathbf{Z}(t)}{\mathrm{d} t}=\mathbf{M Z}(t)-\mu \mathbf{I},
$$

where $\mathbf{Z}(t)=\left(z_{1}(t), \ldots, z_{N}(t)\right)^{T}$ is the vector of trajectories, $\mathbf{I}$ is the $N \times N$ identity matrix, and $\mathbf{M}$ is a tridiagonal matrix with elements $m_{j, j}=\mu+p, m_{j, j+1}=-p$, and $m_{i, j}=0$ otherwise. Upon defining the Laplace transform $\tilde{\mathbf{Z}}(s)=\int_{0}^{\infty} \mathbf{Z}(t) e^{-s t} \mathrm{~d} t$ and the initial values $\mathbf{Z}(t=0)=\left(z_{1}(t=0), \cdots, z_{N}(t=0)\right)^{T}$, Eqs. 228 can be written in the form

$$
s \tilde{\mathbf{Z}}(s)=\mathbf{M} \tilde{\mathbf{Z}}(s)-\frac{\mu}{s} \mathbf{I}+\mathbf{Z}(t=0),
$$

and solved explicitly by first inverting $s \mathbf{I}-\mathbf{M}$ and then calculating the inverse Laplace transform of $\tilde{Z}(s)$. After performing the algebra, the solution to Eqs.28 can be expressed as

$$
\left(z_{j}-R_{N-j}\right)=\sum_{k=0}^{N-j}\left(z_{j+k}(t=0)-R_{N-j-k}\right) \frac{(-p t)^{k}}{k !} e^{(\mu+p) t},
$$

where

$$
R_{k} \equiv 1-\left(\frac{p}{\mu+p}\right)^{k+1}
$$

Upon using $z_{1}(t)$ from Eq. 30 in Eq. 26] we find $G_{0}$ as a function of the $z_{j}$ values implicitly expressed through the starting positions $z_{j}(t=0)$ of the trajectorie:

$$
\begin{aligned}
G_{0}\left(z_{1}, \cdots, z_{N}, t\right)=\exp \left[-\alpha t\left(\frac{p}{\mu+p}\right)^{N}-\right. & \alpha \sum_{k=0}^{N-1} \frac{\left(z_{k+1}(t=0)-R_{N-k-1}\right) p^{k}}{(\mu+p)^{k+1} k !} \\
& \times \gamma[k+1,-(\mu+p) t]] .
\end{aligned}
$$


We are thus left with explicitly determining $z_{j}(t=0)$ as a function of the independent variables $z_{j}$. We do this by inverting Eq.30

$$
\left(z_{j}(t=0)-R_{N-j}\right)=\sum_{k=0}^{N-j}\left(z_{j+k}-R_{N-j-k}\right) \frac{(p t)^{k}}{k !} e^{-(\mu+p) t} .
$$

Using this result in Eq. 33 we find

$$
\begin{aligned}
G_{0}\left(z_{1}, \cdots, z_{N}, t\right)=\exp [-\alpha t & \left(\frac{p}{p+\mu}\right)^{N}-\alpha e^{-(\mu+p) t} \sum_{k=0}^{N-1} \sum_{j=0}^{N-k-1} \frac{p^{j+k} t^{j}}{(\mu+p)^{k+1} k ! j !} \\
& \left.\times\left(z_{j+k+1}-R_{N-j-k-1}\right) \gamma[k+1,-(\mu+p) t]\right] .
\end{aligned}
$$

Finally, since the survival probability is obtained by imposing $z_{\ell}=1$ for all $\ell$, we obtain

$$
\begin{aligned}
S_{1}(t)=\exp [-\alpha t & \left(\frac{p}{p+\mu}\right)^{N} \\
& \left.\quad-\frac{\alpha p^{N} e^{-(\mu+p) t}}{(\mu+p)^{N+1}} \sum_{j=0}^{N-1} \sum_{\ell=0}^{N-1-j} \frac{t^{\ell}(\mu+p)^{\ell}}{j ! \ell !} \gamma[j+1,-(\mu+p) t]\right],
\end{aligned}
$$

which is equivalent to Eq.16. We can now successively determine the dynamics of the probability distribution function conditioned on the absorbing site containing a finite number of particles $n_{N+1} \geq 1$. For $n_{N+1}=1$, the corresponding generating function $G_{1}\left(z_{1}, \cdots, z_{N}, t\right)$ can be obtained from the Master equation for $P\left(n_{1}, \cdots, n_{N+1}=1, t\right)$ :

$G_{1}\left(z_{1}, \cdots, z_{N}, t\right)=\sum_{n_{1}, \cdots n_{N}=0}^{\infty} z_{1}^{n_{1}} \cdots z_{N}^{n_{N}} P\left(n_{1}, \cdots n_{N}, n_{N+1}=1, t\right)$.

Upon summing Eq.23 over all values of $n_{j}$ we find that the dynamics of $G_{1}$ is given by

$$
\frac{\partial G_{1}}{\partial t}=\alpha G_{1}\left(z_{1}-1\right)+\frac{\partial G_{0}}{\partial z_{1}}
$$

where $G_{0}$ is the generating function associated with the adsorbing site having no particles, $n_{N+1}=0$, and where the evolution of the trajectories $z_{j}(t)$ are unchanged from those described by Eqs.27. The solution to Eq.37 can be expressed in the form

$$
G_{1}\left(z_{1}, \cdots, z_{N}, t\right)=\lambda(t) G_{0}\left(z_{1}, \cdots, z_{N}, t\right),
$$

where $\lambda(t)$ obeys

$$
\frac{\mathrm{d} \lambda(t)}{\mathrm{d} t}=\frac{p}{G_{0}} \frac{\partial G_{0}}{\partial z_{1}}
$$


The solution for $\lambda(t)$ turns out to be precisely that given in Eq.1. Similarly, it can be found that the generating function with the constraint $n_{N+1}=j$ is given by

$$
\begin{aligned}
G_{j}\left(z_{1}, \cdots, z_{N}, t\right) & =\sum_{n_{1}, \cdots n_{N}=0}^{\infty} z_{1}^{n_{1}} \cdots z_{N}^{n_{N}} P\left(n_{1}, \cdots n_{N+1}=j, t\right) \\
& =\frac{\lambda(t)^{j}}{j !} G_{0}\left(z_{1}, \cdots, z_{N}, t\right),
\end{aligned}
$$

The survival probability $S_{k}(t)$ is given by $S_{k}(t)=\sum_{j=0}^{k-1} G_{j}(1, \cdots, 1, t)$ and moments of the $k^{\text {th }}$ arrival times, found previously, can also be obtained using Eq.15. In addition, an advantage of the generating function approach is that the particle occupations can also be determined. For example, the mean occupation at site $\ell$, conditioned on exactly $j$ particles having entered site $N+1$ is given by

$$
\begin{aligned}
\left\langle n_{\ell}\left(t \mid n_{N+1}=j\right)\right\rangle & =\sum_{n_{1}, \cdots, n_{N}=0}^{\infty} n_{\ell} P\left(n_{1}, \cdots, n_{N+1}=j, t\right) \\
& =\left.\frac{\lambda(t)^{j}}{j !} \frac{\partial G_{0}\left(1, \cdots, z_{\ell}, \cdots, 1, t\right)}{\partial z_{\ell}}\right|_{z_{\ell}=1} \\
& =-\frac{\lambda(t)^{j}}{j !} e^{-\lambda(t)} \alpha p^{\ell-1} e^{-(\mu+p) t} \sum_{k=0}^{\ell-1} \frac{\gamma[k+1,-(\mu+p) t] t^{\ell-k-1}}{(\mu+p)^{k+1} k !(\ell-k-1) !} .
\end{aligned}
$$

Upon summing Eq.41 over all $j$, we find that the unconditioned mean occupation $\left\langle n_{\ell}(t)\right\rangle$ is given by

$$
\begin{aligned}
\left\langle n_{\ell}(t)\right\rangle & =\sum_{n_{1}, \cdots, n_{N+1}=0}^{\infty} n_{\ell} P\left(n_{1}, \cdots, n_{N+1}, t\right) \\
& =-\alpha p^{\ell-1} e^{-(\mu+p) t} \sum_{k=0}^{\ell-1} \frac{\gamma[k+1,-(\mu+p) t] t^{\ell-k-1}}{(\mu+p)^{k+1} k !(\ell-k-1) !} .
\end{aligned}
$$

Two limits are of interest: the mean occupation conditioned on no particles hitting site $N+1$, which is given by

$$
\left\langle n_{\ell}\left(t \mid n_{N+1}=0\right)\right\rangle=e^{-\lambda(t)}\left\langle n_{\ell}(t)\right\rangle,
$$

and the average occupation of site $N+1$, regardless of the occupation state of all other sites, which is simply $\left\langle n_{N+1}(t)\right\rangle=\lambda(t)$. Thus, in the long time limit, the occupation of the final site $N+1$ will scale as

$$
\left\langle n_{N+1}(t)\right\rangle \sim \alpha t\left(\frac{p}{\mu+p}\right)^{N},
$$

indicating that at site $N+1$ particles accumulate linearly at a rate that is proportional to the injection rate $\alpha$ attenuated by the evaporation probability for each of the $N$ intervening sites. 

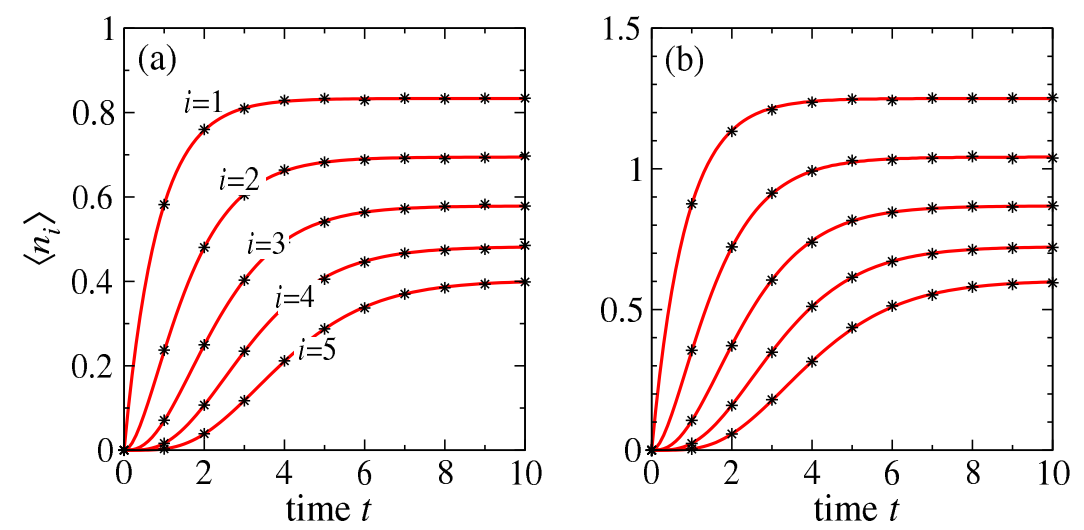

Figure 5. Time dependence of the mean site occupancies. Both panels display exact values (solid lines) from Eq. 43 and simulation (asterisks) for parameter values $p \equiv 1, N=5$, and $\mu=0.2$. Each curve and approximating points correspond to mean occupations at different sites, with earlier site having higher occupations.

In Figure 5, we have plotted the mean occupations derived from Eq. 43 for $N=5$ and $\mu=0.2$. All mean occupancies are seen to reach steady state by $t \approx 10$ and, for all times, mean occupancy is a monotonically decreasing function of site index due to decay. In Fig. 5(a), $\alpha=1<p+\mu$, implying that particles are cleared out faster than they are injected, resulting in $\left\langle n_{1}\right\rangle$ approaching a value less than one. In (b), the $\alpha=1.5>p+\mu$, and $\left\langle n_{1}\right\rangle$ (and $\left\langle n_{2}\right\rangle$ asymptotes to values greater than one. Our results are verified with Monte Carlo simulations.

Finally, the full distribution for $P\left(n_{1}, \cdots, n_{N+1}=j, t\right)$ can be found by using the Cauchy integral [18, over Eq.40.

$P\left(n_{1}, \ldots, n_{N+1}=j, t\right)=\frac{1}{2 \pi i} \oint_{C} \frac{G_{j}\left(z_{1}, \cdots, z_{N}, t\right)}{z_{1}^{n_{1}+1}, \cdots z_{N}^{n_{N}+1}} \mathrm{~d} z_{1} \cdots \mathrm{d} z_{N}$,

where the integral is closed along a path encircling the origin. Evaluating the residues, the above integral can be expressed as

$P\left(n_{1}, \ldots, n_{N+1}=j, t\right)=\left.\frac{\lambda(t)^{j}}{j !} \prod_{\ell=1}^{N}\left(\frac{\partial}{\partial z_{\ell}}\right)^{n_{\ell}} G_{0}\left(z_{1}, \cdots, z_{N}, t\right)\right|_{z_{\ell}=0}$

which can be calculated explicitly to yield

$$
\begin{aligned}
P\left(n_{1}, \ldots, n_{N+1}=\right. & j, t)=\frac{\lambda(t)^{j} G_{0}(0, \cdots, 0, t)}{j !} \\
& \times \prod_{\ell=1}^{N}\left[-\alpha e^{-(\mu+p) t} \sum_{k=0}^{\ell-1} \frac{p^{\ell-1} t^{\ell-1-k}}{(p+\mu)^{k+1} k !} \gamma[k+1,-(\mu+p) t]\right]^{n_{\ell}} .
\end{aligned}
$$




\section{Zero-range Model with Surface Dynamics}

Surface dynamics differs from bulk dynamics in that only the top particle at a given site is able to hop or decay, while the ones below remain inert. The dependencies thus introduced between the particles renders the $k$ th hitting time, and even the survival probability $S_{k}(t)$ of the final site, too difficult to derive in the general $N$ case. In particular, we cannot use the strategy employed for bulk dynamics because it relied essentially on particles injected before time $t$ having independent probabilities of reaching site $N+1$ by time $t$. In the case of surface dynamics, only the top particle in a pile attempts to move to a neighboring pile. The difficulty arises in keeping track of which sites are empty and which ones contain at least one particle.

Beginning with the approach of the previous sub-section, we first consider the Master equation for the distribution of the site occupancies obeying surface dynamics

$$
\begin{aligned}
\dot{P}\left(\left\{n_{\ell}\right\}, t\right)= & -\alpha\left[P\left(\left\{n_{\ell}\right\}, t\right)-P\left(n_{1}-1, \ldots, n_{N+1}, t\right)\left(1-\delta_{n_{1}, 0}\right)\right] \\
& -\mu \sum_{j=1}^{N}\left[\left(1-\delta_{n_{j}, 0}\right) P\left(\left\{n_{\ell}\right\}, t\right)-P\left(n_{1}, \ldots, n_{j}+1, \cdots, t\right)\right] \\
& -p \sum_{j=1}^{N}\left(1-\delta_{n_{j}, 0}\right) P\left(\left\{n_{\ell}\right\}, t\right) \\
& -p \sum_{j=1}^{N}-\left(1-\delta_{n_{j+1,0}}\right) P\left(n_{1}, \ldots, n_{j}+1, n_{j+1}-1, \ldots, t\right) .
\end{aligned}
$$

We now introduce the marginal probability

$$
P_{i}\left(n_{i}, t\right)=\sum_{\left\{n_{j \neq i}\right\}=0}^{\infty} P\left(n_{1}, \ldots, n_{N+1}, t\right),
$$

where the sum is taken over all $n_{j}$ for all sites $1 \leq j \leq N+1$, except site $j=i$. Eq.49 represents the probability that site $i$ has $n_{i}$ particles regardless of the occupation of all other sites. Similarly, the joint probability for sites $i-1$ and $i$ is defined as

$$
P_{i-1, i}\left(n_{i-1}, n_{i}, t\right)=\sum_{n_{j \neq i-1, i}=0}^{\infty} P\left(n_{1}, \ldots, n_{N+1}, t\right) .
$$

Upon summing Eq.48 over all values of $n_{j \neq i}$, we find the time evolution for the marginal probability $P_{i}\left(n_{i}, t\right)$ as a function of the two-site probabilities $P_{i-1, i}\left(n_{i-1}, n_{i}, t\right)$ :

$$
\begin{aligned}
\dot{P}_{i}\left(n_{i}, t\right) \quad= & p \sum_{n_{i-1}=1}^{\infty}\left(1-\delta_{n_{i}, 0}\right) P\left(n_{i-1}, n_{i}-1, t\right)-p \sum_{n_{i-1}=1}^{\infty} P\left(n_{i-1}, n_{i}, t\right)+\quad(51) \\
& +(\mu+p) P_{i}\left(n_{i}+1, t\right)-(\mu+p) P\left(n_{i}, t\right)\left(1-\delta_{n_{i}, 0}\right), \quad 1<i \leq N+1
\end{aligned}
$$

Continuing in this way, the equations for the marginal occupation probabilities form a hierarchy which is completed by the equation for the injection site $i=1$ : 


$$
\begin{aligned}
\dot{P}_{1}\left(n_{1}, t\right)= & -\alpha\left[P_{1}\left(n_{1}, t\right)-P_{1}\left(n_{1}-1, t\right)\left(1-\delta_{n_{1}, 0}\right)\right] \\
& -(\mu+p)\left[P_{1}\left(n_{1}, t\right)\left(1-\delta_{n_{1}, 0}\right)-P_{1}\left(n_{1}+1, t\right)\right] .
\end{aligned}
$$

Note that the dynamics for site $i=1$ is completely decoupled from that of the other sites so that the marginal occupation distribution of the first site can be solved directly. We now consider two cases where analytic results can be found.

\subsection{Single-site ZRP densities and mean first passage times}

Since Eq.53 is decoupled from the hierarchy, we can solve it by taking its Laplace transform and using the initial condition $P\left(n_{1}, 0\right)=\delta_{n_{1}, 0}$ to find

$$
\begin{aligned}
& \tilde{P}_{1}\left(n_{1}=1, s\right)=\frac{s+\alpha}{\mu+p} \tilde{P}_{1}\left(n_{1}=0, s\right)-\frac{1}{\mu+p} \\
& \tilde{P}_{1}\left(n_{1}+1, s\right)=\left(1+\frac{s+\alpha}{\mu+p}\right) \tilde{P}_{1}\left(n_{1}, s\right)-\frac{\alpha}{\mu+p} \tilde{P}_{1}\left(n_{1}-1, s\right) .
\end{aligned}
$$

The solution can be expressed in the form

$$
\tilde{P}_{1}\left(n_{1}, s\right)=\left[\frac{1-z_{1}(s)}{s}\right] z_{1}(s)^{n_{1}},
$$

where

$z_{1}(s)=\frac{1}{2(\mu+p)}\left((s+\alpha+\mu+p)-\sqrt{(s+\alpha+\mu+p)^{2}-4 \alpha(\mu+p)}\right)$.

Upon inverting, we find

$$
z_{1}(t)=\frac{e^{-(\alpha+\mu+p) t} \sqrt{\alpha}}{t \sqrt{\mu+p}} I_{1}(2 \sqrt{\alpha(\mu+p)} t),
$$

where $I_{1}(t)$ is the first-order modified Bessel Function of the first kind. From Eq. 55 and using the fact that the inverse Laplace transform of a product is a convolution in time, we can iteratively construct $P_{1}\left(n_{1}, t\right)$ starting from $P_{1}(0, t)$

$$
P_{1}\left(n_{1}=0, t\right)=1-\int_{0}^{t} z_{1}\left(t^{\prime}\right) \mathrm{d} t^{\prime},
$$

where $z_{1}(t)$ is given by Eq. 57 and

$$
P_{1}\left(n_{1}, t\right)=\int_{0}^{t} P_{1}\left(n_{1}-1, t^{\prime}\right) z_{1}\left(t^{\prime}\right) \mathrm{d} t^{\prime}
$$

The integrals in Eq. 58 and 59 do not have simple closed forms. However, the functions $P_{1}\left(n_{1}, t\right)$ can also be obtained from differentiation using the relation

$$
\begin{aligned}
P_{1}\left(n_{1}+1, t\right)=(1 & \left.-\delta_{n_{1}, 0}+\frac{\alpha}{\mu+p}\right) P_{1}\left(n_{1}, t\right) \\
& +\frac{\dot{P}_{1}\left(n_{1}, t\right)-\alpha P_{1}\left(n_{1}-1, t\right)\left(1-\delta_{n_{1}, 0}\right)}{\mu+p} .
\end{aligned}
$$


For instance, we may recursively write

$$
P_{1}\left(n_{1}=1, t\right)=\frac{\alpha}{\mu+p}\left(1-\int_{0}^{t} z_{1}\left(t^{\prime}\right) \mathrm{d} t^{\prime}\right)-\frac{z_{1}(t)}{\mu+p} .
$$

In the case of $N=1$ we can also solve for the first $(k=1)$ passage times by observing that the equation for the two-site distribution function, conditioned on $n_{2}=0$, is also decoupled from the hierarchy:

$$
\begin{aligned}
\dot{P}\left(n_{1}, 0, t\right)= & \alpha\left[P\left(n_{1}-1,0, t\right)\left(1-\delta_{n_{1}, 0}\right)-P\left(n_{1}, 0, t\right)\right]+\mu P\left(n_{1}+1,0, t\right) \\
& \left.-(\mu+p) P\left(n_{1}, 0, t\right)\right)\left(1-\delta_{n_{1}, 0}\right),
\end{aligned}
$$

where we have dropped the subscripts on the two-site distribution function so that $P\left(n_{1}, n_{2}, t\right) \equiv P_{1,2}\left(n_{1}, n_{2}, t\right)$. Using Laplace transforms, we find

$$
\tilde{P}\left(n_{1}, 0, s\right)=\frac{y_{1}(s)^{n_{1}}}{s+\alpha-\mu y_{1}(s)}
$$

where

$$
y_{1}(s)=\frac{\alpha+\mu+p+s-\sqrt{(\alpha+\mu+p+s)^{2}-4 \alpha \mu}}{2 \mu} .
$$

From this solution of $\tilde{P}\left(n_{1}, n_{2}=0, s\right)$, we can obtain the Laplace transformed probability that site $i=2$ has not been hit by a particle $\tilde{S}_{1}(s)=\sum_{n_{1}=0}^{\infty} \tilde{P}\left(n_{1}, n_{2}=\right.$ $0, s)$. Thus, in the $N=1$ case, the mean first passage time is

$\left\langle T_{1}\right\rangle=\sum_{n_{1}=0}^{\infty} \tilde{P}\left(n_{1}, 0, s=0\right)=\frac{\alpha+\mu+p+\sqrt{(\alpha+\mu+p)^{2}-4 \alpha \mu}}{2 \alpha p}$.

Note that in the case of $\mu=0$, this result simplifies to $\left\langle T_{1}\right\rangle=\alpha^{-1}+p^{-1}$. In surface dynamics without desorption, the first passage time is determined by the dynamics of the lead particle. Therefore, the mean first arrival time is simply the total time it takes for the leading particle to reach site $N+1$ and is given by $\left\langle T_{1}\right\rangle=\alpha^{-1}+N p^{-1}$.

\subsection{Steady-state limit}

We have not been able to find closed-form solutions of the surface dynamics ZRP for general $N$ and nonzero desorption rate $\mu>0$. However, Eq. 53 can be solved in the steady state limit by using the ansatz $P_{1}\left(n_{1}\right)=\left(1-z_{1}\right) z_{1}^{n_{1}}$. The equation supports a solution when $z_{1}=\alpha(\mu+p)^{-1}$, implying

$$
P_{1}\left(n_{1}\right)=\left(1-\frac{\alpha}{\mu+p}\right)\left(\frac{\alpha}{\mu+p}\right)^{n_{1}} .
$$

The above expression is correct only for $\alpha<\mu+p$, so that $0 \leq P_{1}\left(n_{1}\right) \leq 1$. Physically this condition is simply a statement of that if injection is too fast, the occupations 
continues to build without bound. Steady-state occupations arise only if the injection rate $\alpha$ is small enough such that hopping $p$ and evaporation $\mu$ can keep up.

In order to solve Eq.51, we need to a closure relation for the two-site probability distribution $P_{i-1, i}\left(n_{i-1}, n_{i}\right)$. As shown in [5], the two-site probability distribution can be factorised in the steady-state limit and expressed as $P_{i-1, i}\left(n_{i-1}, n_{i}\right)=$ $P_{i-1}\left(n_{i-1}\right) P_{i}\left(n_{i}\right)$. If we impose that each $P_{j}\left(n_{j}\right)$ has a power law dependence in $n_{j}$, similar to what done for $n_{1}$, it is easy to verify that the steady-state marginal probabilities are solved by

$$
P_{j}\left(n_{j}\right)=\left(1-\frac{\alpha p^{j-1}}{(\mu+p)^{j}}\right)\left(\frac{\alpha p^{j-1}}{(\mu+p)^{j}}\right)^{n_{j}}, \quad \alpha<\mu+p .
$$

The resulting steady-state mean occupation at each site are

$$
\left\langle n_{j}\right\rangle=\frac{\alpha p^{j-1}}{(\mu+p)^{j}-\alpha p^{-1}}, \quad \alpha<\mu+p .
$$

From our steady-state results for $N>1$, we can find an approximation to the passage times by a mean-field argument in which the probability of site $N+1$ surviving up to $k-1$ particles hitting it obeys $\dot{S}_{k}(t)=-J(t) S_{k}(t)$. The particle current $J(t)=p \sum_{n_{N}=1}^{\infty} P\left(n_{N} \mid T_{k}>t\right)$ is conditioned on fewer than $k$ particles having arrived at site $N+1$ by time $t$. Since neither $P\left(n_{N} \mid T_{k}>t\right)$, nor the unconditional distribution $P\left(n_{N}, t\right)$ are available, we must approximate $J(t)$ with its steady-state, "mean-field" (single site marginal distribution) value through Eq.66.

$$
\begin{aligned}
J & \approx p \sum_{n_{N}=1}^{\infty} P\left(n_{N}, t \rightarrow \infty\right) \\
& =p \sum_{n_{N}=1}^{\infty}\left(1-\frac{\alpha p^{N-1}}{(\mu+p)^{N}}\right)\left(\frac{\alpha p^{N-1}}{(\mu+p)^{N}}\right)^{n_{N}} \\
& =\frac{\alpha p^{N}}{(\mu+p)^{N}} .
\end{aligned}
$$

In this approximation, $J$ is independent of $k$ and all interarrival times are approximately

$$
\left\langle T_{k}\right\rangle-\left\langle T_{k-1}\right\rangle=\int_{0}^{\infty} S_{k}(t) \mathrm{d} t \approx \frac{(\mu+p)^{N}}{\alpha p^{N}} .
$$

As expected, this estimate is precisely that given by $\alpha_{\text {eff }}$ in Eq.21 for the bulk dynamics case and is accurate in the limit of $\alpha \ll(\mu+p)$ where the entry flux is slow compared to the internal dynamics and the first passage time is dominated by the contribution given by $\alpha^{-1}$. Fast internal dynamics allows the system to quickly reach steady-state, rendering the interarrival times equivalent for bulk and surface dynamics.

Upon taking the limit of slow injection rate $\alpha \rightarrow 0$ in Eq.64 we find

$$
\lim _{\alpha \rightarrow 0}\left\langle T_{1}\right\rangle=\frac{(\mu+p)}{\alpha p},
$$

which is identical to the result in Eq.69 for $N=k=1$. Figure 6 plots simulated interarrival times and compares them with those from bulk dynamics. For large $k$, 


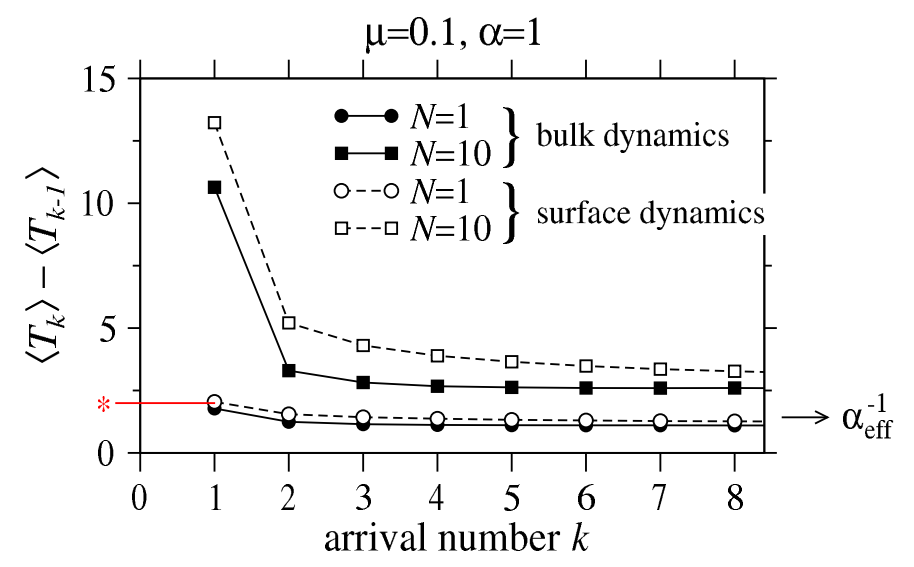

Figure 6. Interarrival times $\left\langle T_{k}\right\rangle-\left\langle T_{k-1}\right\rangle$ for the surface dynamics ZRP as a function of $k$. Results from Monte Carlo simulations (open symbols) for both $N=1$ and $N=10$ are presented. Since fewer particle are mobile in surface dynamics, the arrival times are longer. The simulations match the analytic results found for the $N=k=1$ (Eq.64 indicated by the asterisk) and large $k$ (Eqs. 69 and 22) limits. For comparison numerical results for the bulk dynamics case (filled symbols) are also plotted. All plots were derived using $\mu=0.1$ and $\alpha=1$.

interarrival times for both bulk and surface dynamics approach the same value $\alpha_{\text {eff }}^{-1}$ for each $N$. The only exact result for surface dynamics is that given by Eq. 64 for $N=k=1$, and is indicated by the asterisk at $\left\langle T_{1}\right\rangle \approx 2.05125$.

\section{Summary and Conclusions}

In this paper, we have provided detailed and explicit calculations of first passage times of an $N$-site, one-dimensional Zero-Range Process. Both a Poissonian injection process at an injection site, and spontaneous desorption of all sites were included. We considered both bulk dynamic and surface dynamic rules as illustrated in Fig. 1]

For the ZRP obeying bulk dynamics, we computed the particle passage times using two methods. In the first method, we explicitly enumerated the random walks of each injected particle and evaluated their probability of reaching the final absorbing site within a time window. The probability that the absorbing site has not absorbed more than $k$ particles by a certain time was constructed. The main results for the survival probabilities are given by Eqs.2] and 1, with explicit expressions for the mean first passage time given by Eq.17 and its subsequent asymptotic limits.

We also derived the complete Master equation for the probability distribution for a ZRP obeying bulk dynamics, and solved its corresponding generating function using the method of characteristics. In addition to the $k^{\text {th }}$ passage time distribution, this yielded the mean conditional occupancies of each site given by Eq.41, and the full probability distribution given by Eq.47.

Finally, for a single site $(N=1)$ ZRP obeying surface dynamics, we found exact results for the site density distribution (Eqs. 58 and 59) and the mean first passage times (Eq.64). Note that higher moments of the first passage time are readily obtained by evaluating higher derivatives of Eq.62 at $s=0$. For general $N$, only the steadystate particle currents and interarrival times could be found in closed form (Eq.69). 
The authors thank T. Antal for fruitful discussions. This work was supported by the NSF through grants DMS-0349195 and DMS-0719462 and by the NIH through grant K25AI058672.

\section{References}

[1]Evans M R and Hanney T 2005 Nonequilibrium statistical mechanics of the zero-range process and related models J Phys A: Math. Gen. 38 R195-R240

[2]Evans M R and Braz J 2000 J Phys A: Math. Gen. 30, 42

[3] Grobkinsky S, Schutz G M and Spohn H 2003 J Stat Phys 113, 389 (2003)

[4]Noh J D, Shim G M and Lee H 2005 Complete Condensation in a Zero Range Process on Scale-Free Networks Phys. Rev. Lett. 94198701

[5]Evans M R, Majumdar S N and Zia R K P 2004 actorised Steady States in Mass Transport Models J Phys A: Math. Gen. 37, L275

[6]D'Orsogna M R and Chou T 2009 Optimal transport and apparent drug resistance in viral infections, PLoS One 4 e8165

[7]Gardner A J and Evans J P 2006 Mammalian membrane block to polyspermy: new insights into how mammalian eggs prevent fertilisation by multiple sperm Reprod Fertil Dev 18 53-61

[8]Godrche C and Luck J M 2005 Dynamics of the condensate in zero-range processes J. Phys. A: Math. Gen. 38 7215-7237

[9]Pinedo M L Scheduling: Theory, Algorithms, and Systems (Prentice Hall, New York, 2008).

[10]Redner S A Guide to First-Passage Processes, (Cambridge University Press, Cambridge, 2001).

[11]Angel A G and Zia R K P 2009 Power spectra in a zero-range process on a ring: total occupation number in a segment, J. Stat. Mecg. P03009

[12]Bortz A B, Kalos MH and Leibowitz J L 1975 A New Algorithm for Monte Carlo Simulation of Ising Spin Systems, J. Comp. Phys. 1710

[13] Harris R J, Rakos A and Schütz G M 2005 Current fluctuations in the zero-range process with open boundaries, J. Stat. Mech. P08003

[14] Angel A G, Schmittmann B and Zia R K P 2007 Zero-range process with long-range interactions at a T-junction J. Phys. A: Math. Theor. 40 12811-12828

[15]Lindenberg K, Seshadri V, Shuler K E and Weiss G H 1980 Lattice Random Walks for Sets of Random Walkers: First Passage Times J. Stat. Phys. 23 11-25

[16]Bender C and Orszag S A Advanced Mathematical Methods for Scientists and Engineers: Asymptotic Methods and Perturbation Theory, (Springer-Verlag, New York 1999)

[17]Whitham G B Linear and Nonlinear Waves, (Wiley, New York 1974)

[18]Ahlfors L V Complex Analysis, (McGraw-Hill, New York 1979) 